\title{
Cognitive Assessments in Critical Care Patient Populations: Methodological Considerations
}

\author{
Sachin Agarwal ${ }^{1 *}$ (1) and Marykay Pavol ${ }^{2}$
}

๑ 2021 Springer Science+Business Media, LLC, part of Springer Nature and Neurocritical Care Society

The high incidence of neuropsychological deficits after critical illnesses and their decisive impact on long-term recovery are well described in the literature [1-3]. Early cognitive testing after acute stabilization has been proposed as a method to elucidate risk factors for poor cognitive outcomes and identify opportunities for intervention such as early referral for cognitive therapy. Brief, efficient, and reliable neuropsychological assessment can be useful in clinical practice and research to quantify cognitive functioning in people with acute brain injury following intracranial or systemic insults. The National Institutes of Health Toolbox for the Assessment of Neurological and Behavioral Function Cognition Battery (NIHTB-CB) [4] is a computerized neuropsychological screening battery, which has been recommended as a common data element for brain injury research and clinical trials. Of note, all studies to date have been conducted with patients living in the community [5]. Assessment of hospitalized patients must consider issues related to delirium, ability to adhere to standardized administration, and the timing of examinations in relation to the date of injury/illness onset. Consequently, whereas bedside mental status examinations are commonly performed, formal cognitive assessment is less common in hospitalized patients [5]. Patients in the early stages of

\footnotetext{
*Correspondence: sa2512@columbia.edu

${ }^{1}$ Division of Neurocritical Care and Hospitalist Neurology, Department of Neurology, New York Presbyterian Hospital/Columbia University Irving Medical Center, 177 Ft. Washington Avenue, Floor: 8-300, New York, NY 10032, USA

Full list of author information is available at the end of the article
}

This article is related to the original article available at https://doi. org/10.1007/s12028-020-01126-8.

\section{望 Springer}

recovery from critical illness are at high risk of impairments in attention and stamina.

In the current issue of Neurocritical care, Maas et al. [6] performed a prospective, observational cohort study on 73 patients presenting with acute onset of either intracerebral hemorrhage $(\mathrm{ICH} ; n=38)$ or sepsis $(n=53)$ as representative neurologic and systemic critical illnesses. The NIHTB-CB and Patient-Reported Outcomes Measurement Information System (PROMIS) cognition measures were administered seven days after intensive care unit (ICU) discharge or at hospital discharge, whichever occurred first. PROMIS instruments for patient-reported cognitive evaluations were successfully obtained in 42 ( $58 \%$ overall; $79 \%$ of sepsis and $34 \%$ of $\mathrm{ICH}$ ) patients, and scores were favorable (median was 97th percentile compared to the general adult population). Patients who completed PROMIS cognitive assessments differed from those who did not by having less severe encephalopathy in the early phase of critical illness and at the time of hospital discharge. Testable patients had shorter ICU length of stay, less severe functional impairment at hospital discharge, and a lower proportion of patients had aphasia symptoms, and premorbid history of dementia, compared to those unable to participate. NIHTB-CB tests were obtainable in only 9 (12\% overall) patients, and nonparticipation was deemed not due to the reduced level of consciousness as the majority completed PROMIS assessments and did not differ from untestable patients by level of self-reported cognitive symptoms. Authors concluded that there is a severe discordance in patientreported and objective cognitive assessments among patients in the early recovery phase, suggesting infeasibility, invalidity, and unsuitable nature of cognitive tests that were validated for use in patients with chronic medical and neurologic illnesses. 
As highlighted by the authors, the study had few limitations including small sample size, which was performed in a single center, and the data from two heterogeneous populations were combined, thus reducing the internal validity of the findings; compared to patients admitted with sepsis, ICH patients had worse participation rates.

Some other methodological concerns deserve further discussion. First, to amplify external generalizability and adopt pragmatism, the authors did not use any strict inclusion/exclusion criteria before enrollment. Survival to hospital discharge, absence of alteration in consciousness or delirium at the time of cognitive assessments, and upfront exclusion of patients with a preexisting severe psychiatric or cognitive disorder are typical considerations in critical illness survivorship studies [3, 5, 6]. Including them in the analysis precluded any direct comparisons of the current study findings with others in the literature and more importantly does not support the infeasibility and unsuitability nature of the tests argument concluded by the authors.

Second, the NIHTB-CB, like any tool, is effective and accurate only when used as intended. Thus, there should be no surprise that measures and normative samples designed for community-dwelling patients, when applied to those in the early stages of recovery from critical illness, were found to produce "invalid results." The NIHTB-CB manual comment on the use of the measures with "special" populations (e.g., pediatric, elderly, disabled community-dwelling patients) but provide no guidance on the minimal cognitive skills required for the examination. This is not to suggest that patients in the earliest stages of critical illness recovery are inappropriate for an objective cognitive examination. These patients merit assessment with measures appropriate for their recovery stage and sensitive to change as they progress, possibly through an early cognitive screening program before hospital discharge with outpatient follow-up for detailed assessment. Multiple factors arising from a stay in an ICU setting such as mechanical ventilation, related communication difficulties, the high prevalence of delirium, patient physical, and cognitive fatigue, formal indepth assessment of critically ill patients is challenging. Recovery tends to be more rapid in the first few weeks from the event, and cognition may continue to improve in the first 6 to 12 months after ICU discharge $[3,5,7]$. Patients while returning to their respective outpatient clinics within 1-2 months after hospital discharge for routine follow-up may have reached clinical stability, either recovered from any transient cognitive dysfunction (e.g., delirium, effects of medications) or experienced previously nonexistent functional limitations due to acquired cognitive impairment after they have had a chance to resume their normal daily activities. These routine follow-up visits could be leveraged to achieve reliable and standardized outcome assessment for research purposes and can more easily be integrated into standard medical care.

Finally, the frequency of co-occurrence of functional impairments and emotional disturbances with newly acquired cognitive impairment is high among critical illness survivors and persist beyond acute care hospitalization [8]. Studies looking at the effects of other aspects of survivorship on cognitive outcomes are needed.

The future study will test the feasibility of early cognitive testing and determine barriers and facilitators associated with the implementation of a cognitive screening program in a mixed-methods approach with input from various stakeholders. By this method, the challenges in testing ICU survivors in the early recovery period, as demonstrated by Maas et al., could be uncovered and tweaked to address specific stages of recovery.

\section{Author details \\ ${ }^{1}$ Division of Neurocritical Care and Hospitalist Neurology, Department of Neu- rology, New York Presbyterian Hospital/Columbia University Irving Medical Center, 177 Ft. Washington Avenue, Floor: 8-300, New York, NY 10032, USA. \\ ${ }^{2}$ Department of Neurology and Rehabilitation and Regenerative Medicine, Columbia University Irving Medical Center, New York, USA.}

\section{Acknowledgements}

Dr. Agarwal is supported by the National Heart, Lung, And Blood Institute of the National Institutes of Health (Grant No. R56 HL153311).

Conflicts of interest

Authors have no conflict of interest.

\section{Publisher's Note}

Springer Nature remains neutral with regard to jurisdictional claims in published maps and institutional affiliations.

Received: 29 December 2020 Accepted: 31 December 2020

Published online: 20 January 2021

\section{References}

1. Stienen MN, Visser-Meily JMA, Schweizer TA, Hänggi D, Macdonald RL, Vergouwen $\mathrm{MDI}$, et al. Prioritization and timing of outcomes and endpoints after aneurysmal subarachnoid hemorrhage in clinical trials and observational studies - proposal of a multidisciplinary research group. Neurocrit Care. 2019;30:102-13.

2. Hemphill JC, Farrant M, Neill TA. Prospective validation of the ICH Score for 12-month functional outcome. Neurology. 2009;73:1088-94.

3. Needham DM, Davidson J, Cohen $\mathrm{H}$, et al. Improving long-term outcomes after discharge from the intensive care unit: Report from a stakeholders' conference. Crit Care Med. 2012;40:502-9.

4. Weintraub S, Dikmen SS, Heaton RK, Tulsky DS, Zelazo PD, Bauer PJ, Carlozzi NE, Slotkin J, Blitz D, Wallner-Allen K, Fox NA, Beaumont JL, Mungas D, Nowinski CJ, Richler J, Deocampo JA, Anderson JE, Manly JJ, Borosh B, Havlik R, Conway K, Edwards E, Freund L, King JW, Moy C, Witt E, Gershon RC. Cognition assessment using the NIH Toolbox. Neurology. 2013;80(11 Suppl 3):S54-64. 
5. Pavol MA. Inpatient neuropsychological assessment in older adults. In: Ravdin LD, Katzen HL, editors. Handbook on the neuropsychology of aging and dementia. Cham: Springer International Publishing; 2019. p. 89-103.

6. Maas MB, Lizza BD, Kim M, Gendy M, Liotta EM, Reid KJ, Zee PC, Griffith $J W$. The feasibility and validity of objective and patient-reported measurements of cognition during early critical illness recovery. Neurocrit Care. 2020.
7. Iwashyna TJ, Ely EW, Smith DM, Langa KM. Long-term cognitive impairment and functional disability among survivors of severe sepsis. JAMA. 2010;304(16):1787-94.

8. Marra A, Pandharipande PP, Girard TD, Patel MB, Hughes CG, Jackson JC, Thompson JL, Chandrasekhar R, Ely EW, Brummel NE. Co-occurrence of post-intensive care syndrome problems among 406 survivors of critical illness. Crit Care Med. 2018;46(9):1393-401. 